\title{
mTORC1 Overactivation Leads to Abnormalities in Skeletal Development
}

\author{
Sayuki Iwahashi, ${ }^{a, \#}$ Kazuya Tokumura ${ }^{a, \#}$ Gyujin Park,${ }^{a}$ Shinsuke Ochiai, ${ }^{a}$ Yasuka Okayama ${ }^{a}$ \\ Hiroki Fusawa, ${ }^{a}$ Kaname Ohta ${ }^{a}$ Kazuya Fukasawa, ${ }^{a}$ Takashi Iezaki, ${ }^{a}$ and Eiichi Hinoi $*, a, b$ \\ ${ }^{a}$ Laboratory of Pharmacology, Department of Bioactive Molecules, Gifu Pharmaceutical University; Gifu 501-1196, Japan: \\ and ${ }^{b}$ United Graduate School of Drug Discovery and Medical Information Sciences, Gifu University; Gifu 501-1196, Japan. \\ Received August 3, 2020; accepted September 6, 2020
}

The mechanistic/mammalian target of rapamycin complex-1 (mTORC1) integrates multiple signaling pathways and regulates various cellular processes. Tuberous sclerosis complex 1 (Tsc1) and complex 2 (Tsc2) are critical negative regulators of mTORC1. Mouse genetic studies, including ours, have revealed that inactivation of mTORC1 in undifferentiated mesenchymal cells and chondrocytes leads to severe skeletal abnormalities, indicating a pivotal role for mTORC1 in skeletogenesis. Here, we show that hyperactivation of mTORC1 influences skeletal development through its expression in undifferentiated mesenchymal cells at the embryonic stage. Inactivation of $T s c 1$ in undifferentiated mesenchymal cells by paired-related homeobox 1 (Prxl)-Cremediated recombination led to skeletal abnormalities in appendicular skeletons. In contrast, Tsc1 deletion in chondrocytes using collagen type II $\alpha 1$ (Col2a1)-Cre or in osteoprogenitors using Osterix (Osx)-Cre did not result in skeletal defects in either appendicular or axial skeletons. These findings indicate that Tsc complexmediated chronic overactivation of mTORC1 influences skeletal development at the embryonic stage through its expression in undifferentiated mesenchymal cells but not in chondrocytes or osteoprogenitors.

Key words skeletogenesis; undifferentiated mesenchymal cell; tuberous sclerosis complex 1; mechanistic/ mammalian target of rapamycin complex-1 (mTORC1)

\section{INTRODUCTION}

During embryogenesis, skeletons are generated by two distinct ossification processes, designated intramembranous ossification and endochondral ossification in mammals. ${ }^{1)}$ In the endochondral ossification process, mesenchymal stem cells undergo differentiation into chondrocytes to form a cartilaginous template, which is subsequently replaced with bone that contains bone-forming osteoblasts and bone-resorbing osteoclasts. $^{2)}$ In the intramembranous ossification process, mesenchymal stem cells directly differentiate into osteoblasts. ${ }^{3)}$

The mechanistic/mammalian target of rapamycin (mTOR) is an evolutionarily conserved serine/threonine kinase that forms two different complexes designated mTOR complex-1 (mTORC1) and mTORC2. ${ }^{4)}$ mTORC1 contains the regulatoryassociated protein of mTOR (Raptor) subunit, and regulates various cellular processes, including growth, proliferation, differentiation, survival, and autophagy. ${ }^{5)}$ Tuberous sclerosis complex $1(T s c 1)$ and complex $2(T s c 2)$, also known as hamartin and tuberin, negatively regulate $\mathrm{mTORC} 1{ }^{6}{ }^{6}$ Recent mouse genetic studies indicated that $\mathrm{mTORC} 1$ contributes to the pathogenesis of various diseases, including cancer, diabetes, aging, cardiac disease, and neurological diseases, as well as in a variety of physiological functions. ${ }^{7}$

Using cell-specific disruption of the Raptor gene, we have recently demonstrated that expression of mTORC1 in undifferentiated mesenchymal cells and chondrocytes plays a critical role in skeletogenesis. For example, conditional ablation of the Raptor gene using paired-related homeobox 1 (Prxl)-Cre-mediated recombination, which occurred in undifferentiated mesenchymal cells, led to a severe impairment of appendicular skeletal formation. ${ }^{8)}$ Moreover, chondrocyte-specific Raptor

\footnotetext{
\# These authors contributed equally to this work.

* To whom correspondence should be addressed. e-mail: hinoi-e@gifu-pu.ac.jp
}

deficient embryos using collagen type II $\alpha 1$ (Col2al)-Cre-dependent recombination displayed a very severe and generalized chondrodysplasia. ${ }^{8)}$ Although several independent lines of evidence based on genetic strategies show that inactivation of mTORC1 signaling leads to abnormal skeletal development in vivo, the effect of sustained overactivation of mTORC1 on skeletal development is largely unknown. ${ }^{9,10)}$ In this study, we generated and analyzed mutant embryos that harbor conditional deletion of the Tscl gene, a negative regulator of mTORC1, in undifferentiated mesenchymal cells, chondrocytes, or osteoprogenitors to further establish the role of mTORC1 in skeletal development.

\section{MATERIALS AND METHODS}

Mice $T_{s c l^{f l f l}}$ mice were crossed with either Prxl-Cre, Col2al-Cre, or Osterix (Osx)-Cre mice. ${ }^{11-13)}$ These mutant mice were backcrossed for more than five generations with C57BL/6J. Osx-Cre mice were maintained in the absence of doxycycline. The study protocol meets the guidelines of the Japanese Pharmacological Society and was approved by the Committee for Ethical Use of Experimental Animals at Gifu University and Gifu Pharmaceutical University. Genotyping was performed by PCR with specific primers.

Skeletal Preparation and Histological Analyses Skeletal preparation was performed as described previously. ${ }^{14)}$ In brief, embryos fixed with $95 \%$ ethanol were immersed in Alcian blue solution, and subsequently stained in Alizarin red solution. The skeletons were then cleaned with $1 \% \mathrm{KOH} / 20 \%$ glycerol. Mouse tibiae were fixed with $10 \%$ formalin, and subsequently embedded in paraffin, followed by section at a thickness of $5 \mu \mathrm{m}$. Sections were stained with hematoxylin \& eosin, von Kossa, Safranin O, and Alcian blue.

Culture of Primary Chondrocytes and Immunoblotting 
(A)

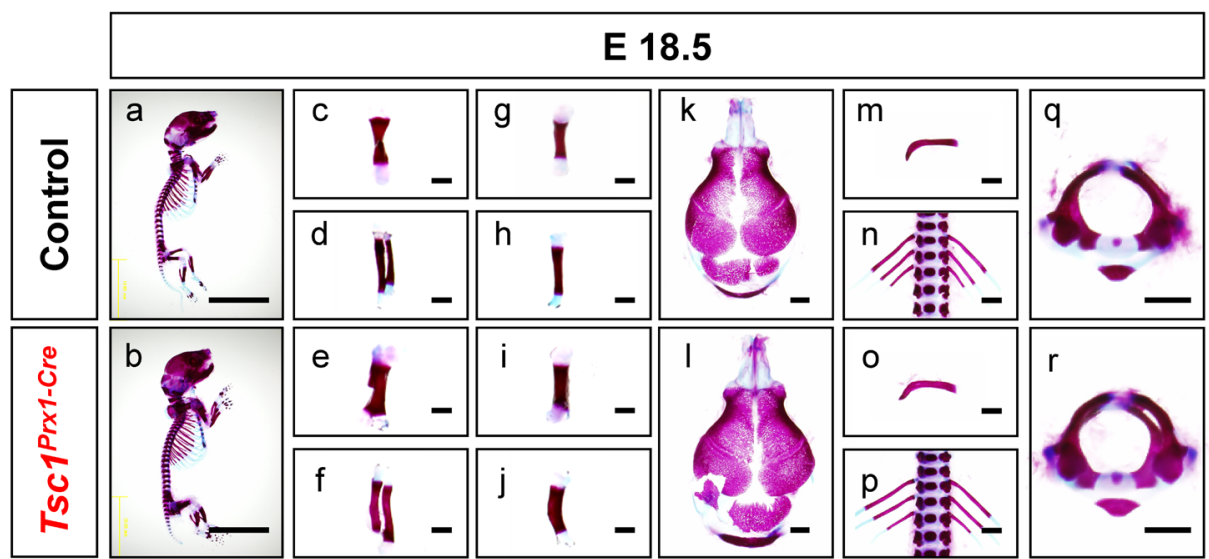

(B)

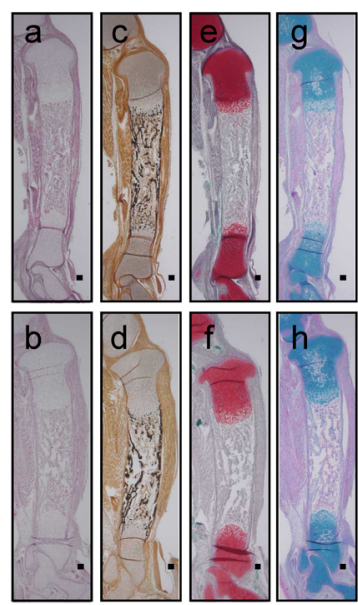

(C)

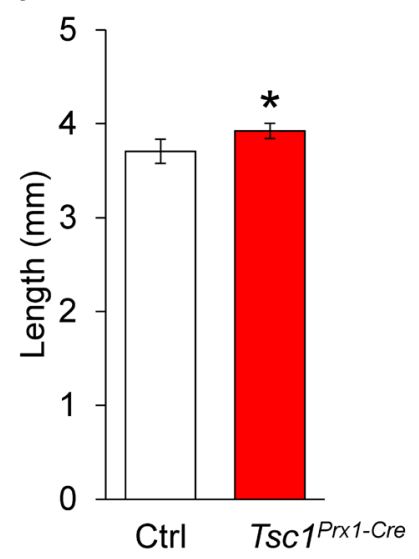

(D)

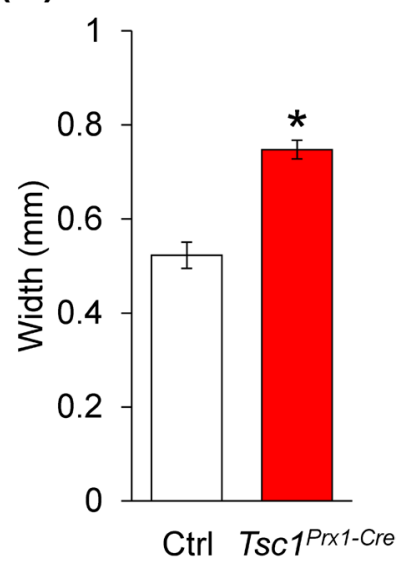

Fig. 1. mTORC1 Activation in Undifferentiated Mesenchymal Cells Leads to Skeletal Abnormalities

(A) The whole and parts of skeleton of Prxl-Cre; $T s c l^{f / f}$ embryos at E18.5. Embryos were double stained with alizarin red and alcian blue. Bar $=10 \mathrm{~mm}(\mathrm{a}, \mathrm{b})$ and Bar $=1 \mathrm{~mm}(\mathrm{c}-\mathrm{r})$. (B) Histological analyses of the tibiae at E18.5. Tibiae were stained with Hematoxylin and Eosin (H\&E), von Kossa, safranin O, and alcian blue (a-h) Bar $=100 \mu \mathrm{m}$. Quantitative data of (C) length and (D) width of long bones of Prxl-Cre;Tsc flift embryos at E18.5 ( $n=10-12)$. Representative images of skeletal preparations and histological analyses derived from more than 3 embryos from different litters are shown. $* p<0.05$, significantly different from the value obtained in control embryos. (Color figure can be accessed in the online version.)

Analysis Primary costal chondrocytes were prepared from new born mice using a sequential enzymatic digestion method as described previously. ${ }^{15}$ ) Cells were solubilized in lysis buffer containing protease inhibitor cocktail, and then samples were subjected to immunoblotting assay. ${ }^{16)}$ Antibodies were from the following companies: anti- $\beta$-tubulin was from Santa Cruz Biotechnology (Santa Cruz, CA, U.S.A.); anti-Tsc1 and anti-p-p70S6K1 were from Cell Signaling Technology (Danvers, MA, U.S.A.).

Data Analysis All results were expressed as the mean \pm standard error of the mean. Statistical significance was determined using the two-tailed, unpaired Student's $t$-test and one-way ANOVA with Bonferroni post hoc test.

\section{RESULTS}

Tsc1 Deficiency in Undifferentiated Mesenchymal Cells Leads to Skeletal Abnormalities To investigate whether mTORC1 hyperactivation could affect skeletal development in vivo, we first inactivated the $T s c 1$ gene in undifferentiated mesenchymal cells using Prxl-Cre transgenic mice. Staining of skeletal preparations from embryos at E18.5 with alcian blue and alizarin red indicated that the gross skeletal anatomy

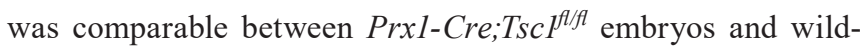
type (WT) embryos at this age (Figs. 1Aa, b). However, long bones in the forelimbs (humerus, radius, and ulna) and hindlimbs (femur and tibia) were larger in Prxl-Cre;TscI $P^{f / f l}$ embryos, and a smaller space was observed in the interfrontal suture (the space between the frontal bones) (Figs. 1Ac-1). On the contrary, the clavicle, whole vertebrae, atlas $(\mathrm{C} 1)$, and axis (C2) of Prxl-Cre;Tscl $f^{f l f l}$ embryos were indistinguishable from those of WT embryos at E18.5 (Figs. 1Am-r). Histological analyses also revealed that both the length and width of these bones were significantly longer in Prxl-Cre; $T_{s c 1} 1^{f / f l}$ embryos at E18.5 compared with those in WT embryos (Figs. 1B-D).

Collectively, these results indicate that constitutive overactivation of mTORC1 in undifferentiated mesenchymal cells leads to skeletal abnormalities at the embryonic stage.

Tsc1 Deficiency in Chondrocytes or Osteoprogenitors Does Not Lead to Skeletal Abnormalities We next investigated whether hyperactivation of $\mathrm{mTORC1}$ in chondrocytes or osteoprogenitors could affect skeletal development. To that end, the $T s c 1$ gene was deleted using either recombination with Col2al-Cre, specific for chondrocytes, or Osx-Cre, specific for osteoprogenitors. Skeletal preparations indicated that the gross structure was comparable between Col2al- 
(A)

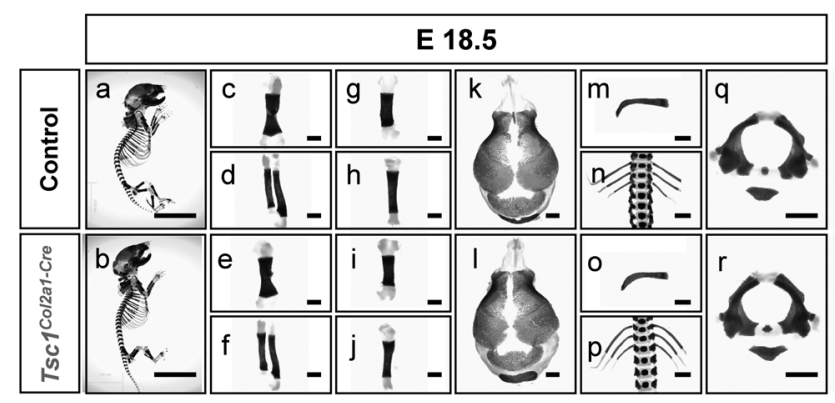

(E)

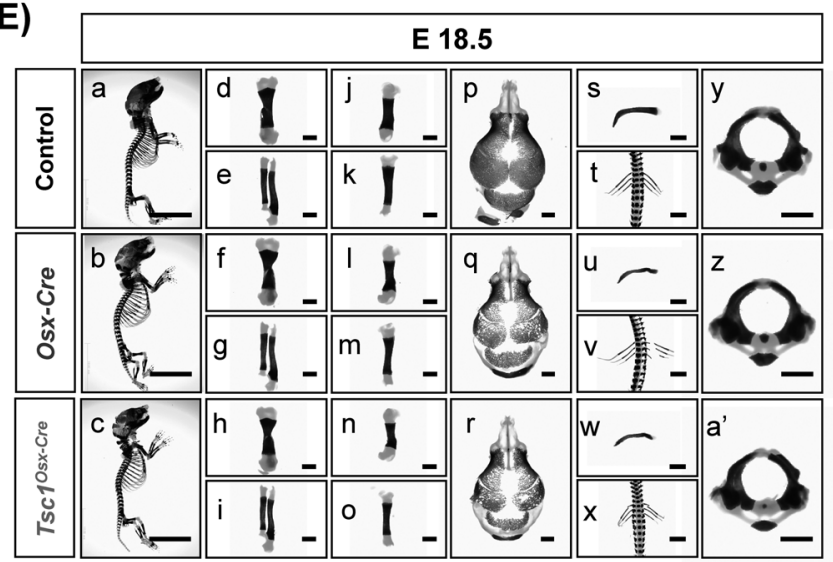

(B)

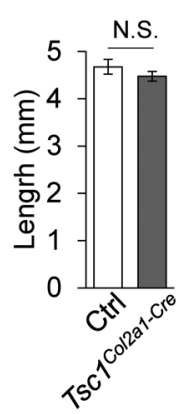

(C)

(D)
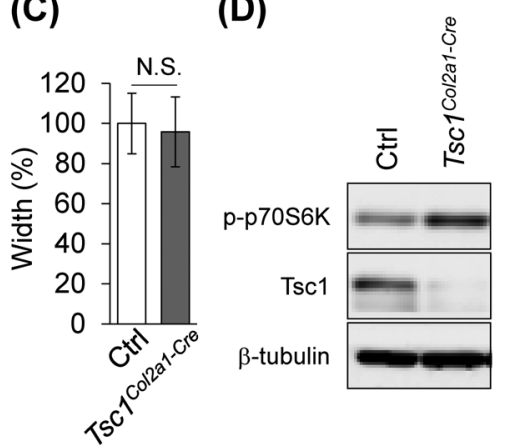

(F)

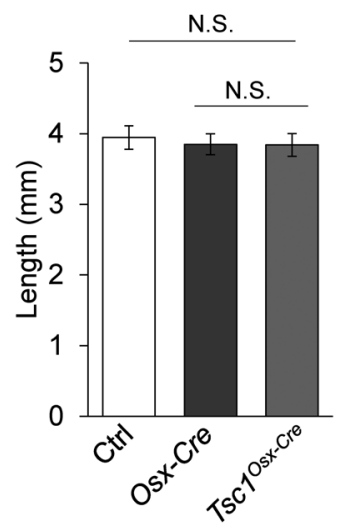

(G)

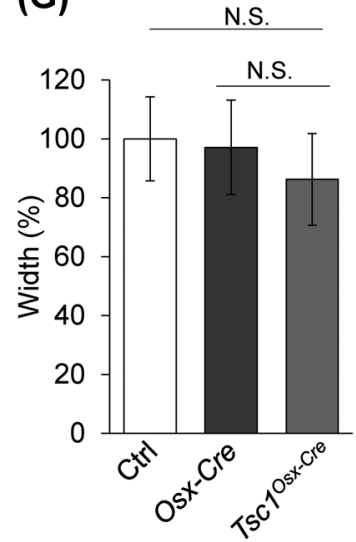

Fig. 2. mTORC1 Activation in Chondrocytes or Osteoprogenitors Does Not Lead to Skeletal Abnormalities

(A) The whole and parts of skeleton of Col2al-Cre; Tscl fl/f embryos at E18.5. Embryos were double stained with alizarin red and alcian blue. Bar = 10 mm (a, b) and $\mathrm{Bar}=1 \mathrm{~mm}(\mathrm{c}-\mathrm{r})$. Quantitative data of (B) length and (C) width of long bones of Col2al-Cre;Tsc1 $1^{f / f}$ embryos at E18.5 $(n=4)$. (D) Tsc1 expression and phosphorylation level of p70S6K in chondrocytes of Col2al-Cre;Tsc fllft mice $(n=3)$. (E) The whole and parts of skeleton of Osx-Cre;Tscl/lfl and Osx-Cre embryos at E18.5. Embryos were double stained with alizarin red and alcian blue. Bar $=10 \mathrm{~mm}(\mathrm{a}-\mathrm{c})$ and Bar $=1 \mathrm{~mm}\left(\mathrm{~d}-\mathrm{a}^{\prime}\right)$. Quantitative data of $(\mathrm{F})$ length and $(\mathrm{G})$ width of long bones of $O s x$-Cre; TscI $P^{f / f l}$ and Osx-Cre embryos at E18.5 $(n=6-10)$. Representative images of skeletal preparations derived from more than 3 embryos from different litters are shown. N.S., not significant.

Cre; $T s c f^{f l / f}$ embryos and WT embryos at E18.5 (Figs. 2Aa, b). In addition, no marked skeletal abnormalities were observed in the long bones, calvaria, clavicle, or vertebrae of Col2alCre; $T_{S c} f^{f l f t}$ embryos at E18.5 (Figs. 2Ac-r). Histological analyses also revealed that both the length and width of these bones did not significantly differ between mutant and WT embryos (Figs. 2B, C). In the cultured chondrocytes of Col2alCre; $T s c f^{f / f l}$ mice, Tsc1 expression was markedly abolished and the level of phosphorylated p70S6K was increased, indicating an increase in mTORC1 activity in chondrocytes (Fig. 2D).

Because the Osx-Cre transgene itself caused development defects in bones formed by the intramembranous ossification process, but not by the endochondral ossification process, Osx-Cre embryos were also used as a control for the effects of Osx-Cre recombination. ${ }^{17)}$ No gross differences were seen between Osx-Cre;Tscl $P^{f l / l}$ embryos, Osx-Cre embryos, and WT embryos at E18.5 (Figs. 2Ea-c). Skeletal elements formed by endochondral ossification, including forelimbs (humerus, radius, and ulna), hindlimbs (femur and tibia), and vertebrae, appeared to be normal in Osx-Cre;Tscl $f^{f / f l}$ embryos (Figs. 2Ed-o, $\mathrm{t}, \mathrm{v}, \mathrm{x}$, and $\left.\mathrm{y}-\mathrm{a}^{\prime}\right)$. Indeed, no significant alternations in the lengths and widths of bones were observed in Osx-Cre;Tscl $\mathrm{P}^{\mathrm{flfl}}$ embryos when compared with Osx-Cre embryos or WT embryos (Figs. 2F, G). Although Osx-Cre;Tsc $f^{f / f l}$ embryos and Osx-Cre embryos at E18.5 exhibited skeletal hypoplasia in the calvaria and clavicle, which are formed by intramembranous ossification and likely arose due to the effect of Osx-Cre recombination itself, no marked skeletal abnormalities were seen in these structures of Osx-Cre;Tscl fl/fl embryos when compared with Osx-Cre embryos (Figs. 2Ep-s, u, w).

Collectively, these results indicate that the hyperactivation of mTORC1 in chondrocytes or osteoprogenitors has a less significant impact on skeletal development at the embryonic stage.

\section{DISCUSSION}

Mouse genetic studies, including our own work, have recently revealed the critical role of $\mathrm{mTORC1}$ in skeletal development during embryogenesis, including by disrupting the Raptor gene via Prxl-Cre or Col2al-Cre to inactivate mTORC1 activity in undifferentiated mesenchymal cells or chondrocytes. ${ }^{8,9)}$ Moreover, it has been shown that hyperactivation of $\mathrm{mTORC1}$ in undifferentiated mesenchymal cells or chondrocytes mediates postnatal skeletal development. In that study, Prx1-Cre;Tsc $1^{f l f l}$ mice displayed shorter and thicker long bones with increased bone volume, but decreased bone mineralization at the adolescent stage, suggesting that mTORC1 overactivation via Prxl-Cre-mediated recombination disrupted postnatal bone quality (size and mass). ${ }^{18)}$ Further, Col2al-

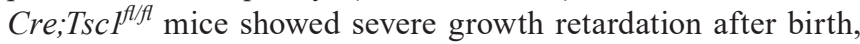
characterized by hypertrophy of growth plate chondrocytes, or 
disorganization in the resting zone without affecting hypertrophy or proliferation, suggesting that mTORC1 overactivation via Col2al-Cre-mediated recombination disrupted postnatal bone development. ${ }^{19,20)}$ However, to the best of our knowledge, this is the first study to demonstrate that hyperactivation of mTORC1 mediates skeletal development during embryogenesis through its expression in undifferentiated mesenchymal cells but not in chondrocytes or osteoprogenitor cells.

Because Prxl-Cre targets the mesenchymal cells in the limb buds but not in the vertebrae, ${ }^{11)}$ the skeletal abnormalities of Prxl-Cre;Tscl $P^{\text {flfl }}$ embryos were observed only in the long bones. Moreover, the fact that both the length and width of the long bones were significantly greater in Prxl-Cre;Tscl $P^{\text {flf }}$ embryos indicates that the mutant embryos display an acceleration of endochondral ossification because endochondral ossification leads to growth in both length and width of long bones. ${ }^{1)}$

Inactivation of mTORC1 activity by conditional ablation of the Raptor gene in undifferentiated mesenchymal cells or chondrocytes resulted in severe impairment of skeletal formation or severe chondrodysplasia at the embryonic stage. ${ }^{8)}$ Together with these previous findings, our current study indicates that both inactivation and overactivation of mTORC1 lead to developmental abnormalities in some skeletal compartments, suggesting that a complex regulatory system may be required to control $\mathrm{mTORC1}$ activity in undifferentiated mesenchymal cells for the maintenance of proper skeletal development during embryogenesis. Although further studies should be performed to reveal the precise mechanisms by which mTORC1 hyperactivation in undifferentiated mesenchymal cells influences skeletal development at the embryonic stage, our work contributes to an improved understanding of the molecular mechanisms underlying skeletal development. Further, these findings may aid in the future development of drugs targeting cartilage diseases associated with the abnormal activation or inactivation of mTORC1 signaling.

Acknowledgments This work was supported in part by the Japan Society for the Promotion of Science (16H05131, 17KT0051, and 18H04971 to E.H.); and the Japan Agency for Medical Research and Development (17824969 to E.H.).

Conflict of Interest The authors declare no conflict of interest.

\section{REFERENCES}

1) Karsenty G, Kronenberg HM, Settembre C. Genetic control of bone formation. Annu. Rev. Cell Dev. Biol., 25, 629-648 (2009).

2) Johnson RL, Tabin CJ. Molecular models for vertebrate limb development. Cell, 90, 979-990 (1997).

3) Hall BK, Miyake T. The membranous skeleton: the role of cell condensations in vertebrate skeletogenesis. Anat. Embryol., 186, 107-124 (1992)

4) Sabatini DM. Twenty-five years of mTOR: Uncovering the link from nutrients to growth. Proc. Natl. Acad. Sci. U.S.A., 114, 1181811825 (2017).
5) Brown EJ, Albers MW, Shin TB, Ichikawa K, Keith CT, Lane WS, Schreiber SL. A mammalian protein targeted by G1-arresting rapamycin-receptor complex. Nature, 369, 756-758 (1994).

6) Nojima H, Tokunaga $\mathrm{C}$, Eguchi S, Oshiro N, Hidayat S, Yoshino K, Hara K, Tanaka N, Avruch J, Yonezawa K. The mammalian target of rapamycin (mTOR) partner, raptor, binds the mTOR substrates p70 S6 kinase and 4E-BP1 through their TOR signaling (TOS) motif. J. Biol. Chem., 278, 15461-15464 (2003).

7) Saxton RA, Sabatini DM. mTOR signaling in growth, metabolism, and disease. Cell, 168, 960-976 (2017).

8) Iezaki T, Horie T, Fukasawa K, Kitabatake M, Nakamura Y, Park G, Onishi Y, Ozaki K, Kanayama T, Hiraiwa M, Kitaguchi Y, Kaneda K, Manabe T, Ishigaki Y, Ohno M, Hinoi E. Translational control of Sox 9 RNA by mTORC1 contributes to skeletogenesis. Stem Cell Reports, 11, 228-241 (2018).

9) Chen J, Long F. mTORC1 signaling controls mammalian skeletal growth through stimulation of protein synthesis. Development, 141, 2848-2854 (2014).

10) Jiang $\mathrm{M}, \mathrm{Fu} \mathrm{X}$, Yang H, Long F, Chen J. mTORC1 Signaling promotes limb bud cell growth and chondrogenesis. J. Cell. Biochem., 118, 748-753 (2017).

11) Logan M, Martin JF, Nagy A, Lobe C, Olson EN, Tabin CJ. Expression of Cre Recombinase in the developing mouse limb bud driven by a Prxl enhancer. Genesis, 33, 77-80 (2002).

12) Terpstra L, Prud'homme J, Arabian A, Takeda S, Karsenty G, Dedhar S, St-Arnaud R. Reduced chondrocyte proliferation and chondrodysplasia in mice lacking the integrin-linked kinase in chondrocytes. J. Cell Biol., 162, 139-148 (2003).

13) Rodda SJ, McMahon AP. Distinct roles for Hedgehog and canonical Wnt signaling in specification, differentiation and maintenance of osteoblast progenitors. Development, 133, 3231-3244 (2006).

14) Iezaki T, Fukasawa $K$, Horie T, Park G, Robinson S, Nakaya M, Fujita H, Onishi Y, Ozaki K, Kanayama T, Hiraiwa M, Kitaguchi Y, Kaneda K, Yoneda Y, Takarada T, Guo XE, Kurose H, Hinoi E. The MAPK Erk5 is necessary for proper skeletogenesis involving a Smurf-SmadSox9 molecular axis. Development, 145, dev164004 (2018).

15) Iezaki T, Ozaki K, Fukasawa $K$, Inoue M, Kitajima S, Muneta $T$, Takeda S, Fujita H, Onishi Y, Horie T, Yoneda Y, Takarada T, Hinoi E. ATF3 deficiency in chondrocytes alleviates osteoarthritis development. J. Pathol., 239, 426-437 (2016).

16) Horie $T$, Park G, Inaba $Y$, Hashiuchi $E$, Iezaki T, Tokumura $\mathrm{K}, \mathrm{Fu}$ kasawa K, Yamada T, Hiraiwa M, Kitaguchi Y, Kamada H, Kaneda K, Tanaka T, Inoue H, Hinoi E. MAPK Erk5 in leptin receptorexpressing neurons controls body weight and systemic energy homeostasis in female mice. Endocrinology, 160, 2837-2848 (2019).

17) Wang L, Mishina Y, Liu F. Osterix-Cre transgene causes craniofacial bone development defect. Calcif. Tissue Int., 96, 129-137 (2015).

18) Wu H, Wu Z, Li P, Cong Q, Chen R, Xu W, Biswas S, Liu H, Xia X, Li S, Hu W, Zhang Z, Habib SL, Zhang L, Zou J, Zhang H, Zhang W, Li B. Bone size and quality regulation: concerted actions of mTOR in mesenchymal stromal cells and osteoclasts. Stem Cell Reports, 8, 1600-1616 (2017).

19) Yan B, Zhang Z, Jin D, Cai C, Jia C, Liu W, Wang T, Li S, Zhang H, Huang B, Lai P, Wang H, Liu A, Zeng C, Cai D, Jiang Y, Bai $\mathrm{X}$. mTORC1 regulates PTHrP to coordinate chondrocyte growth, proliferation and differentiation. Nat. Commun., 7, 11151 (2016).

20) Newton PT, Xie M, Medvedeva EV, Sävendahl L, Chagin AS. Activation of mTORC1 in chondrocytes does not affect proliferation or differentiation, but causes the resting zone of the growth plate to become disordered. Bone Rep., 8, 64-71 (2018). 